\title{
MARS AEROBOT VALIDATION PROGRAM
}

\author{
V.V.Kerzhanovich (1), J.Cutts (1), A.Bachelder (1) J.Cameron (1), J.Hall ${ }^{(1)}$, J.Patzold (1), M.Quadrelli \\ (1) , A.Yavrouian (1), J.Cantrell ${ }^{(2)}$, T.Lachenmeier ${ }^{(3)}$, M.Smith ${ }^{(4)}$
}

\begin{abstract}
The Mars Balloon Validation Program (MABVAP) was initiated in August 1997 to develop and validate key technologies needed for aerobot missions on Mars. The major elements of the program are the development of balloons for flight on Mars, robust techniques for deployment and inflation and modeling and simulation of balloon flight paths. selection, development and tests of available balloon materials, design and fabrication of balloons (both superpressure and solar- heated), design and fabrication of deployment and inflation systems for aerial deployment, design and fabrication of avionics to control deployment/inflation process and to get telemetry and video data. Modeling of main processes during deployment and actual flight is also a part of MABVAP.
\end{abstract}

In order to validate deployment and inflation, MABVAP applies experience from previous Mars balloon development or study activities the Russian-French Mars Aerostat Project (19881995), Mars Aerial Platform Sudy (1994) and Mars Aerobot/Balloon Study (1996). The progran includes laboratory, wind munel, vacuum chamber tests of the system components and a number of tropospheric and stratospheric flight tests of deployment and inflation of lightfilm balloons in a simulated Martian environment

Key issues in the design include: the use of proven materials or their combinations; the availability of adequate balloon fabrication technologies and processes; evacuation of gas from the balloon prior to packaging and the design of a balloon container capable of storing the balloon over a wide range of ambient

(1) Jet Propulsion Laboratory, California Institute of Technology, Pasadena, CA

(2) Space Dynamics Laboratory. Utah State University, Logan, UT

(3) GSSL. Inc., Hillsboro, OR

(4) Raven Industries. Sulphur Springs, TX pressures. During the deployment process several considerations come into play: mitigation of forces on the balloon during deployment; a safe deployment process which does not tear the balloon; avoiding subsequent instabilities of the balloon including helium gas bulb propagation inside the balloon. Other key design issues include the necessity for a reefing mechanism for ensuring stable and predictable inflation of the balloon and the choice between top and bottom inślation.

Tesis that have been, made at JPL and at the Vertical Wind Tunnel at NASA Langley Research Center clarified many of the first order issues discussed above and lead to the baseline configuration with inflation from the bottom without a reefing mecrianism. The system is to be stable during the most critical part of inflation process. Although oscillations may develop when the balloon is filled more than $-60 \%$ in volume the bottom inflation configuration ens'ures that these do not damage the balloon. Free drop tests of the inflated balloon at LARC and at JPL confirmed stability in the free flight. To avoid possible damage of the balloon during inflation. a special diffuser/windsock has been designed to operate at high inflation rates. The system has been validated by inflating $12.5 \mathrm{mk}$ and $8.5 \mathrm{mk} 10-\mathrm{m}$ diameter balloons in the vacuum chamber at 5 mbar ambient pressure.

A series of free flight tests of a brassboard deployment module from an altitude of $1.5 \mathrm{~km}$ have been conducted with the payload dropped initially from a hot air balloon and in later flights from a helicopter. These tests were successful and demonstrated the shock mitigation approach and the stability of inflation of the balloon from gas cylinders beneath the balloon.

The first successful stratospheric tests with simulation of Martian environment using a carrier balloon to raise the deployment module to $\sim 35 \mathrm{~km}$ was performed in March 1999.. Plans are being initiated for tests of a miniaturized system that would be compatible with the payload envelope for a Mars Micromission 
launched by Ariane 5. Development and stratospheric test of this miniaturized system could be conducted during the spring of 1999.

\section{INTRODUCTION}

Airborne platforms and lighter-than-air vehicles specifically may become powerful tools in the future of the planetary exploration. After real experience with the Sojoumer rover and few years of detailed development of powerful rovers for Mars 2003 and 2005 missions became clear strong restrictions of the surface rover's mobility. Probably the realistic travel distances would be limited by tens of kilometers per year at relatively obstacle-free plains and units of kilometers or less at the rugged terrains. Large areas of Mars are inaccessible for rovers at all. LTA should survive deployment loads, it should be inflated safely in a few minutes From this point of view attractiveness of the aerial vehicles is obvious. However the fundamental problem for r.em is low density of the Martian atmosphere that is equivalent to the stratosphere of Earth above $35 \mathrm{~km}$. As it is well known that no heavier-than-air vehicle reached this altitude so far. Low pressure and high thermal contrasts provide a difficulty and for lighter-then-air vehicles (LTA): they must have a considerable size to carry significant payloads.

It would be probably hard to find sites on Mars to la:-nch LTA safely from the surface with existiig technology; the aerial launch i.e. deplcyment and inflation of LTA during the descent in the atmosphere seems now the most feasib'e option. Aerial deployment and inflation has its own problems: LTA should be densely packed and stored during the interplanetary flight, it should survive deployment loads, it should be inflated safely in a few minutes. At present there is no relevant experience of deployment large thin-film inflatable structures in the stratosphere. The study of his process, design work and tests should precede to any Martian LTA missions. Development of technology of the aerial deployment and inflation of thin-film LTA in Martian environment is one of strategic objectives of MABVAP.

\section{BACKGROUND}

The first practical concept for a balloon mission in the atmosphere of Mars was initiated in April 1986 by French and Soviet researchers. The concept was based on the success of the first planetary balloon mission - the Venus VEGA Balloons of 1985. The VEGA balloon heritage significantly influenced the Mars Aerostat approach specifically with respect to the mode of deployment of balloon. A number of studies and tests have been performed that clarified various aspects of the Mars Aerostat performance. The Planetary Society participated in this program with Soviets and French, and developed the concept of the snake or guiderope - which enabled the balloon to tolerate diurnal descents to the surface as the balloon cooled at night and permitted a mission duration of about 10 days as well as in situ surface data.

However the most critical phase - aerial deployment and inflation of a thin-film balloon in the Martian-like environment has not been demonstrated (1). Due to budget problems the Russian-French development was terminated in 1995.

The new concepts - the Mars Aerial Platform (MAP) proposal to the NASA Discovery program in $1994 \quad(2)$ and the Mars Aerobot/Balloon Study (MABS), carried out for NASA in 1996 (3), focused on the use of superpressure balloon with its potential for longduration mission of up to 90 days. The MABS study involved collaboration with the NASA stratospheric balloon research program at NASA's Wallops Flight Facility (WFF) and in a fine example of spin-off from space programs played a role in the formulation of NASA's Earth's Ultra-Long Duration Balloon oroject by WFF. But again the deployment and inflation of the ballocn was not addressec in detail.

Lighter-than-air planetary missions continued aitract growing interest due to unique combination of proximity to the surface and mobility that far surpasses capability of surface vehicles. However, with the success of the Sojourner microrover on the Mars Pathfinder mission in the summer of 1997, JPL was asked by NASA to assess the feasibility of a balloon mission that was an order of magnitude smaller than the Mars Aerostat and these other concepts. The conclusion of this study was that such a mission would be feasible but would require the use of advanced light-weight materials to improve the useful payload as a fraction of floating mass at Mars. In addition, bottominflation - introduction of gas from beneath the balloon was determined to be the most effective way of improving the useful payload as a fraction of the total injected mass at Mars. Thus not only was validation of aerial deployment and 
inflation of a thin-film light-gas balloon an enabling technology for this generation of the planetary missions but it required an approach that has not been well investigated previously.

\section{MARS AEROBOT TECHNOLOGY EXPERIMENT (MABTEX)}

The MABTEX is conceived as the smallest possible long duration Mars Aerobot with a meaningful scientific payload. Recent progress in microminiaturization -Sojourner $(10 \mathrm{~kg})$ and Muses-C $(1.2 \mathrm{~kg})$ rovers, DS-2 Mars Microprobes $(3.5 \mathrm{~kg})$ - lowers the "scientifically useful " payload mass to $2.5-3 \mathrm{~kg}$. The smallest superpressure balloon to carry $2.5 \mathrm{~kg}$ in Martian atmosphere is $10-11 \mathrm{~m}$ sphere. With some margin 0.25-0.3 mil Mylar film can support daytime superpressures when the balloon is heated predominantly by IR-radiation from the surface. Considerations of stability and system mass lead to selection of a bottom inflation concept.

Various approaches to deploying MABTEX to Mars have been considered but at this time there is not a firm plan for a mission. One possible approach has been as a New Millennium technology experiment. More recently, the concept of Mars Micromissions capable of delivering payloads of 30 to $40 \mathrm{~kg}$ into the Mars environment has been introduced. The decision by NASA to carry out a Mars Micromission airplane experiment in 2003 has remced prospects of flying a ballor,in at that opportunity. However, it has drawn great attention to the value of airbome science at Mars and this will benefit the balloon approach when scientsats became more aware of the severe limited lifetimes and data retum constraints of heavierthan-air vehicles at Mars.

\section{MARS AEROBOT VALIDATION}

$$
\text { PROGRAM }
$$

The Mars Balloon Validation Program (MABVAP) was initiated in August 1997 and was conceived as a low-cost earth-based program to be completed prior to carrying out MABTEX. There are three major components to MABVAP: validation of aerial deployment and inflation, superpressure balloon design and development of new simulation tools. The primary emphasis to date and in this paper was on the first - the most critical part. Progress is now being extended to the other two areas. The speed of progress in the MABVAP has been and continues to be limited by budget constraints.
Approach: Our approach to validation of deployment and inflation of a Mars balloon consisted of three phases which became progressively more costly to implement. This approach was devised after extensive consultation with the balloon community and in particular with the CNES team that developed and tested the Mars Aerostat:

Phase 1 - Concept Development; In this first phase, the intent was to explore configuration options, to test innovative concept in the laboratory and in wind tunnels at small scale and to establish the design space by using similarity methods.

Phase 2 - Static Demonstration: In this phase, functionally performing systems were built and static tests were conducted at full scale. This step confirmed the design choices made on the smaller scale and built confidence in the performance in the dynanic environment.

Phase 3 - Dynamic Validation: The goal of this phase was to demonstrate and validate the design approach used in the closest approximation to the environment of Mars- the Earth's stratosphere. Free flight tests performed at low altitude in the Earth's troposphere were not only valuable in demonstrating the functionality of the test systems prior to conducting stratospheric tests but demonstrated the feasibility of the bottom inflation approach for missions to planets with dense atmospheres and particularly Venus and Titan.

We drew on the experience of many other projects. Many individuals and organizations supported MABVAP: French CNES (J.Blamont, A.Vargas, J.Evrard) shared results of Mars Aerostat project, participated in our tests and performed deployment simulations for us, SouthWest Research Institute (B.Perry) described the approach of OBJS project, NASA Centers Langley (R.Whipple, M.Fremaux), Dryden (J. Baer-Riedhard. J.Bauer, M.Rivas), Lewis (J.Carek) provided critical test support, discussions with S.Smith (NASA WFF) and $J . R a n d$ (Winzen Engineering) were important in the test definition. N.Kjomi (University of Wisconsin), D.Nitsche, B.Barry contributed greatly in the stratospheric tests. We enjoyed the support of the FAA. FCC and local authorities of County of Hawaii and BLM for permits of the flight tests. 
Specific Objectives The specific objectives of the program were:

- to define the flight system configuration for the stratospheric flight tests and for MABTEX

- to design, fabricate and test the elements of the test flight system

- to develop models for simulation of essential processes

- to test the most critical phases of the deployment and inflation process

- to reveal and mitigate possible instabilities and hazards

- to perform tropospheric deployment and inflation of a thin-film light-gas balloon to simulate deployment and inflation in the atmosphere of Venus

- to perform stratospheric deployment and inflation of a full scale thin-film light-gas balloon in Mars-like environment

Proslem Areas: Critical issues for aerial
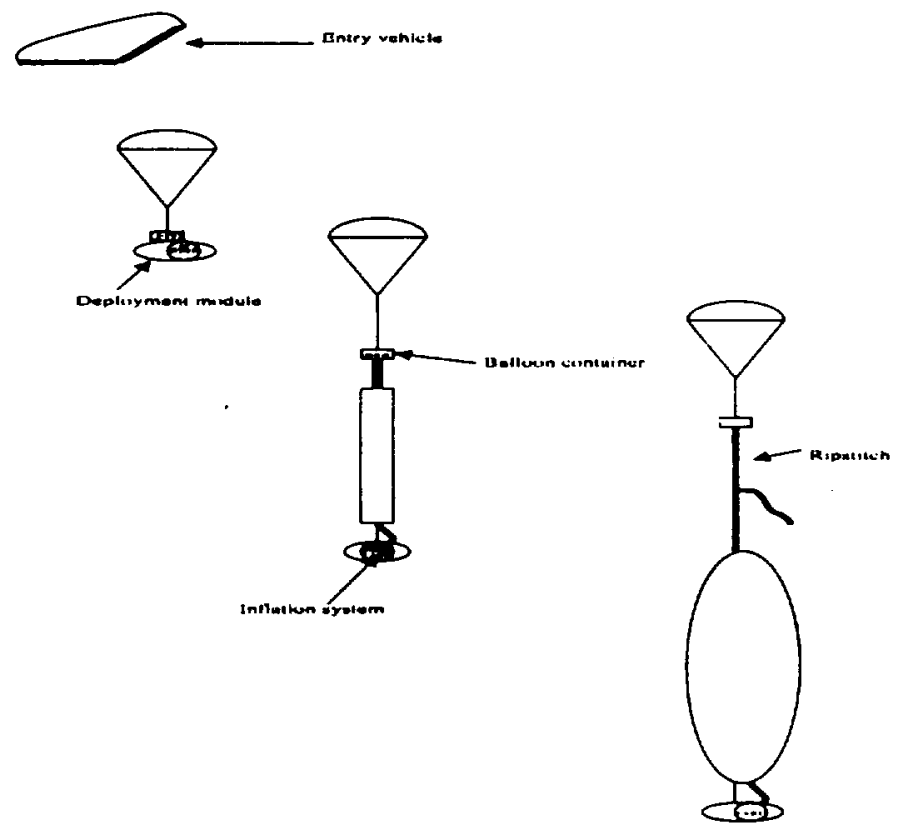

Fig 1 Deployment and Inflation Concept

deployment and inflation were: mitigation of deployment shocks: possible twisting of the balloon; possible "spinnaker" and other aerodynamic instabilities; avoiding if possible a need for reefing the balloon; propagation of the helium bubbie to the top of the balloon when inflated from the bottom; possible damage of the balloon during helium inflation with a rapidly expanding helium (expansion rate $-22 \mathrm{~m}^{3} / \mathrm{s}$ ); residual gas evacuation: compact packaging of the spherical balloon; balloon design for the airborne deployment

Some of the problems were first approached analytically and where that proved insufficient, an extensive test program was developed to study them. The program had a condensed schedule: the wind tunnel tests started in late December 1997, the first attempt of a stratospheric test was in early October 1998.

\section{CONCEPT DEVELOPMENT}

Following extensive evaluation of previous aerial deployment and inflation concepts we adopted a bottom inflation concept and initiated analytical studies, laboratory tests and wind tunnel and free flight tests of smai! scale models to confirm its feasibility. 
Mitigating balloon envelope stresses during deployment: If no restraint mechanisms (such as a braking wheel) are used, the balloon starts a free fall when it deploys from its container (Fig $\mathrm{N})$. Abrupt deceleration of the inflation system suspended from the bottom of the balloon by the balloon envelope produces a shock load at the end of the deployment that would destroy the envelope.

Several approaches to shock mitigation were investigated. In addition to a braking wheel, a combination of a damping device (an elastic tether or a ripstitch shock absorber) with the balloon reinforcement (load lines, polar cups) can be used to mitigate the deployment shock. The ripstitch appears to be more reliable though less precise. Elastic materials do not attenuate the motion rapidly enough and may also become brittle at Martian temperatures. Thus, the ripstitch was used as a prime shock absorber in all our drop tests.

A simple simulation has been performed to estimate required ripping force and length of the ripstitch. A more precise model developed by CNES showed that a short shock with amplitude few times more than the ripping force of the ripstitch may occur during a few milliseconds after stretching the balloon.

To rersice risk of damajing of the envelope we adoptrs a new methos of packaging oi the balloon when the balkson unrolls during the deployinent. The final shock occurs closer to the middle of the balloon where it is taken by much more amount of film than near the fittings. The 1 -m diameter cups made of few layers of mylar were part of the balloon structure. No special load tapes were used; 1" 2 mil mylar tapes used for sealing the balloon provided an additional reinforcement. At the drop tests of real $10-m$ balloon an additional short ripstitch was installed between the bottom fitting and inflation system.

Stability of balloon in bottom inflation configuration: The CNES experience with the Mars Aerostat indicated that the balloon could become unstable when descending under the parachute. The bottom-inflated configuration appeared to offer a significant advantage in that there was no large mass interposed between the parachute and the balloon. We undertook to verify this in a series of tests in a vertical wind tunnel at Langley Research Center (LaRC) and in free flight tests of subscale balloons and parathutes at JPl. I.alRe and at the GSSL ficilily on (sregon. Far application (1) the Mars calse, ill tess depended an similarity scaling to the dymamic conditions that would be cxperienced in stratospheric deployment. Shapes and stability of the balloon were investigated for bottom intlation at the wide range of dynamic pressures; secondary lasks were estimates of drag of partially inflated balloon and study of deployment with elastic tethers

Wind tunnel tests were performed in the vertical wind tunnel at NASA Langley Research Center. We used a spherical Mylar balloon 2-m diameter attached to the floor of the tunnel. $1.5-\mathrm{m}$ diameter parachute was attached to the top fitting of the balloon. The balloon was pre-inflated to $20-100 \%$ of its volume. The tests were performed at the air velocities from 2.5 to $7 \mathrm{~m} / \mathrm{s}$ that corresponds to dynamic pressures from $\sim 4$ to $29 \mathrm{~Pa}$. The typical shape of the balloon during the tasts is shown on Figure 2

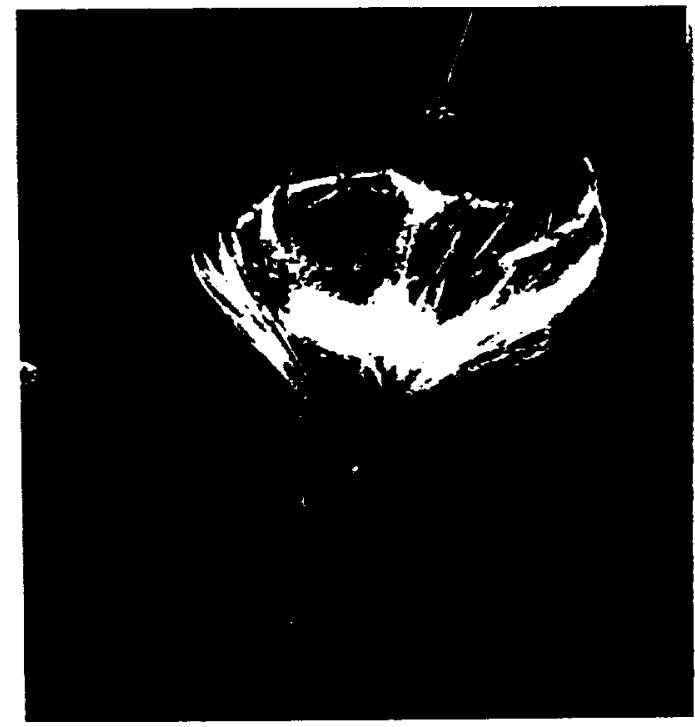

Fig 2. Balloon Test in the LaRC Verticle Wind Tunnell

The main results of these tests were:

- The balloon assumes a "mushroom" shape and remains remarkably stable when inflated less thatn $-50 \%$ at the whole range of dyamic pressures

- He hallewn does not have a tendency to twist

- the avillations induced by von Karman wortces are debeluping when the balloon is matlated more than for\%. their trequency is wase w the extimate from the Struhal mumber 
Free Descent Tests: A few drop tests of a partially inflated balloon suspended under the parachute were performed at LaRC. Though the parachute had a significant gliding angle and the
The specific configurations varied according to the test objectives.

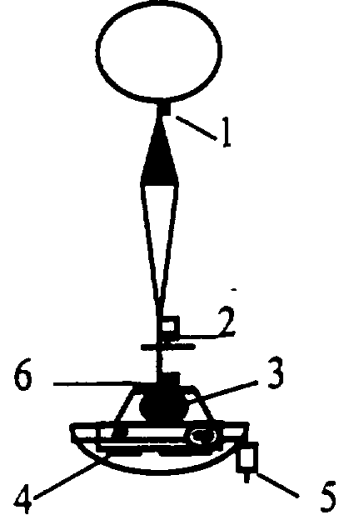

a

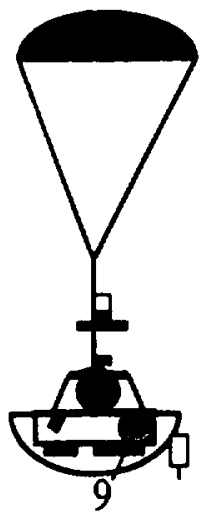

b

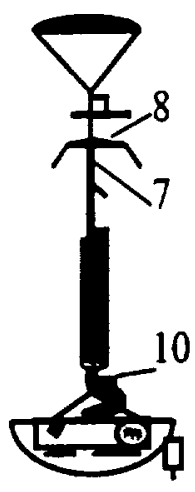

$\mathrm{C}$

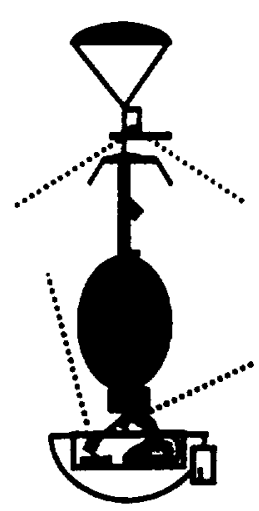

d

Fig 3: Configuration of prototype deployment and inflation test.

I-Pyro-A, 2 - Camera-2, 3 - packed balloon, 4-Camera-1, 5 - radiosonde, 6-Pyro-2, 7 -

ripstitch, 8-top plate, 9-inflation tank, 10-Pyro-3; dashed lines show cameras' field-of-vikw

balloon took a "spinnaker" shape no instabilities were observed. Based on these results, we concluded that a reefing system was not needed for the balloon and all subsequent tests to date have confirmed the validity of this initial conclusion.

Descent tests of it 2-m balloon were alsc performed at JPL ard at the GSSL Tillamook facility to evaluate the development of the oscillations induced by von Karman vortices and the balloon performance. It is appeared that the oscillations during the free descent, though visible, need a time to develop and were much less pronounced than observed in the wind tunnel.

\section{PROTOTYPE SYSTEM DESIGN}

Based on the conceptual development activities, a design for a prototype deployment and inflation system that would be used in static tests and in dynamic tests in the troposphere and stratosphere was developed.

Prototype Flight System: The basic design and test sequence of the system that was used during the flight tests is shown on Fig.3.
Tow vehicle: A manned hot-air balloon and a helicopter were used to lift the flight system at the low-altitude tests; high-altitude carrierballoon was used in the stratospheric tests.

Parachute: flat disk parachutes were used in the most of the flight :ests; these parachutes reliably inflate at the stratosphere

sipstitch: $20-\mathrm{m}$ long webbing folded in the middle and stitched with a certain rows of threads was used as a deployment shock absorber. Number of rows determines a ripping iorce.

Test balloon: Polyethylene balloon simulators with a central load line were used in the lowaltitude deployment tests; Mylar balloons used in inflation and stratospheric tests

Balloon container: a solid box was used as the balloon container the low-altitude tests; a flexible fabric bag is the baseline for the stratospheric tests

Inflation System: The inflation system was designed to deliver helium with the maximum rate $-20 \mathrm{~g} / \mathrm{sec}$. It included two Department of Transportation (DoT) approved tanks, pressure 
regulator, solenoid valve, pressure and temperature transducers, isolation and relief valve. The flexible hose connected system with the diffuser that was a part of the bottom fitting of the balloon. The diffuser was based on design provided by CNES but scaled to a $20 \mathrm{~g} / \mathrm{s}$ inflation rate.

The maximum capacity of the inflation system is $0.9 \mathrm{~kg}$ of helium at 300 atmospheres. The inflation system was adjusted to complete inflation in $-64 \mathrm{sec}$. At an ambient pressure of $5 \mathrm{mb}, 20 \mathrm{~g} / \mathrm{s}$ will result in a volume rated of flow of $22 \mathrm{~m}^{3}$. To reduce the risk of damage to the envelope, a flexible fabric diffuser or "windsock" made of nylon with $-50 \%$ porosity was attached to the output of the metal diffuser. This windsock distributed gas more uniformly. Several inflation tests were conducted in a low pres:"re chamber at JPL in order to optimize the design of the windsock (length and mode of attach ient).

Flight Test Controller: A Flight Test Controller (FTC) that was located in DM commanded the test. The RF command from the ground initiated the preprogrammed test sequence. The set of sensors including a load cell, 3-axis accelerometer, pressure, temperature and GPS.

Deployment module: The whole system was integr:ied as the Deployment Module (DM). A set of carefully adjusted kevlar tethers and bridles controlled the desired configuration on each pnase of the flight test.

Pyro-actuators: Four pyrotechnical cable-cutters were used to execute the flight sequence:

- Pyro-A releases the flight system from the tow-vehicle

- Pyro-1 cuts bridles holding the balloon container and DM together, opens the container and releases the balloon to deploy;

- Pyro-2 separates the test balloon from the parachute

- Pyro-3 cuts the inflation hose and a tether between the test balloon and DM and releases the test balloon for a free flight.

Pyro-A and Pyro-2 were actuated by a RFcommand from FTC over the short-range RF link; it was allowed to avoid potential problems with electrostatic discharge; elimination of long wires in or over the test balloon envelope significantly reduced the risk of its damage.
Videocameras: onc camera (upward looking) was installed in the DM, the other (downward looking) -under the parachute. The cameras provided video coverage of the test sequence.

Long-range video was provided by DRFC to document tests from the ground.

\section{STATIC VALIDATION}

Static Deployment Test: Few static deployment tests were performed at JPL. The critical static deployment test of the real $10-\mathrm{m} 0.5$ mil Mylar balloon has been performed in the $60-\mathrm{m}$ tall hangar of the GSSL Tillamook facility. The ripstitch has been attached to the hangar structure; $35 \mathrm{~kg}$ payload, attached to the bottom fitting of the balloon, simulated DM. The test was successful; the inspection did not find any damages of the balloon. The ripstitch was torn $20 \%$ less than predicted.

The result of another test at GSSL was maintaining of stability of the parachute descent with a very small payload. This test related to the situation during the stratospheric test when drag of the inflated balloon can exceed drag of the parachute. The concern was that the parachute may collapse and may fall on the balloon. The test was performed with 5.4-m diameter flat-disk parachute and the payload $2.5 \mathrm{~kg}$. The parachute inflated and descended steadily.

Static Full-Size Inflation Tests: To validate the overall inflation performance. iwo full scale 10$\mathrm{m}$ sipherical balloons made of Mylar 0.5 and 0.3 mii built by GSSL Inc., were inflated with the inflation system in the environmental thamber. The Space Power Facility of Lewis Research Center (LeRC), now the Glenn Research Center, in Sandusky, OH was used for this purpose. For this test, the top fitting of the ballıon was attached with an elastic tether to the crane at the ceiling; the bottom fitting was attached to the platform. The balloon was stretched in the vertical direction. The inflation system was commanded remotely. Only $0.450 \mathrm{~g}$ of helium was needed to fill the $10 \mathrm{~m}$ diameter $\left(523 \mathrm{~m}^{3}\right.$ balloon at $5 \mathrm{mb}$ and ambient temperature of near $290 \mathrm{~K}$

Inflation of both balloons was completed successfully in $\sim 30$ seconds. The gas expanding through the windsock pushed out the envelope and caused the windsock to move chaotically during its inflation but it did not touched and hence did not damaged the envelope. Fig. 4 shows four stages of the test. 


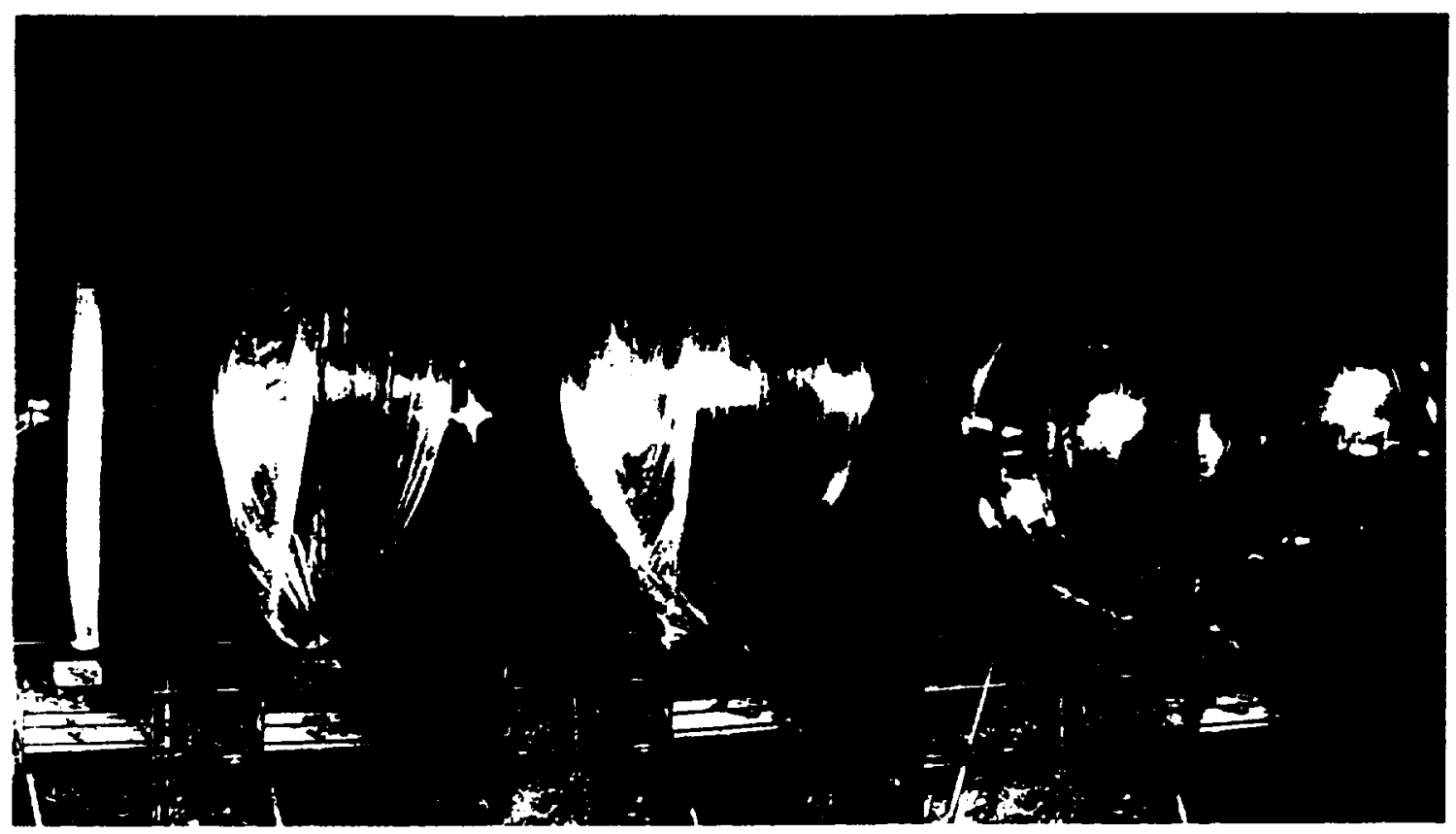

Fig 4: Inflation Test at Lewis Research Center

\section{TROPOSPHERIC TESTS}

Two low altitude tests (LAT-1 and LAT-2) of full-scale deployment system were conducted from either hot air balloon or helicopter from approximately $1500 \mathrm{~m}$ altitude at sites of the Southern California desert near Dryden Flight Resejich Center. The functional aspect: of the basic test system have already been discribed altho:igh the detailed configurs:ion evolved in each iest. The first two tests focused on the deployment system. In both tests $16-\mathrm{m}$ of polyethylene balloon simulator with a central nylun line was packed inside the container. A Deployment Module (DM) $-36 \mathrm{~kg}$ simulated the inflation system. This was followed by LAT-3 a full-up test of deployment and inflation conducted on August 21, 1998.

Deployment System Demonstration in Low Altitude Test 2 (LAT-2) The first test (LAT-1) was performed to verify the performance of the key systems elements and diagnostics; full-scale deployment test LAT 2 was carried out July 21. 1998. This test of the deployment system was highly successful.

The main results were:

- Flight validation of the deployment concept and the packaging scheme
- Flight validatio: of efficiency of the ripstitch to decrease the deployment shock to the acceptable level

- Absence of hazardous rotation of DM or twisting of the envelope after the deployment

- Validation of performance of Flight Test Controller and avionics, RF-pyro actuators and pyro-circuits.

In:ages of the payload at the moment of deployment appear in Fig 5. Fig 5.a was taken from the ground and shows the moment when the balloon was separated from DM and the recovery parachute deployed after Pyro-3 firing. Fig.5.b is taken from the on-board camera during LAT-1 and shows the moment when the balloon starts to deploy from the container. 

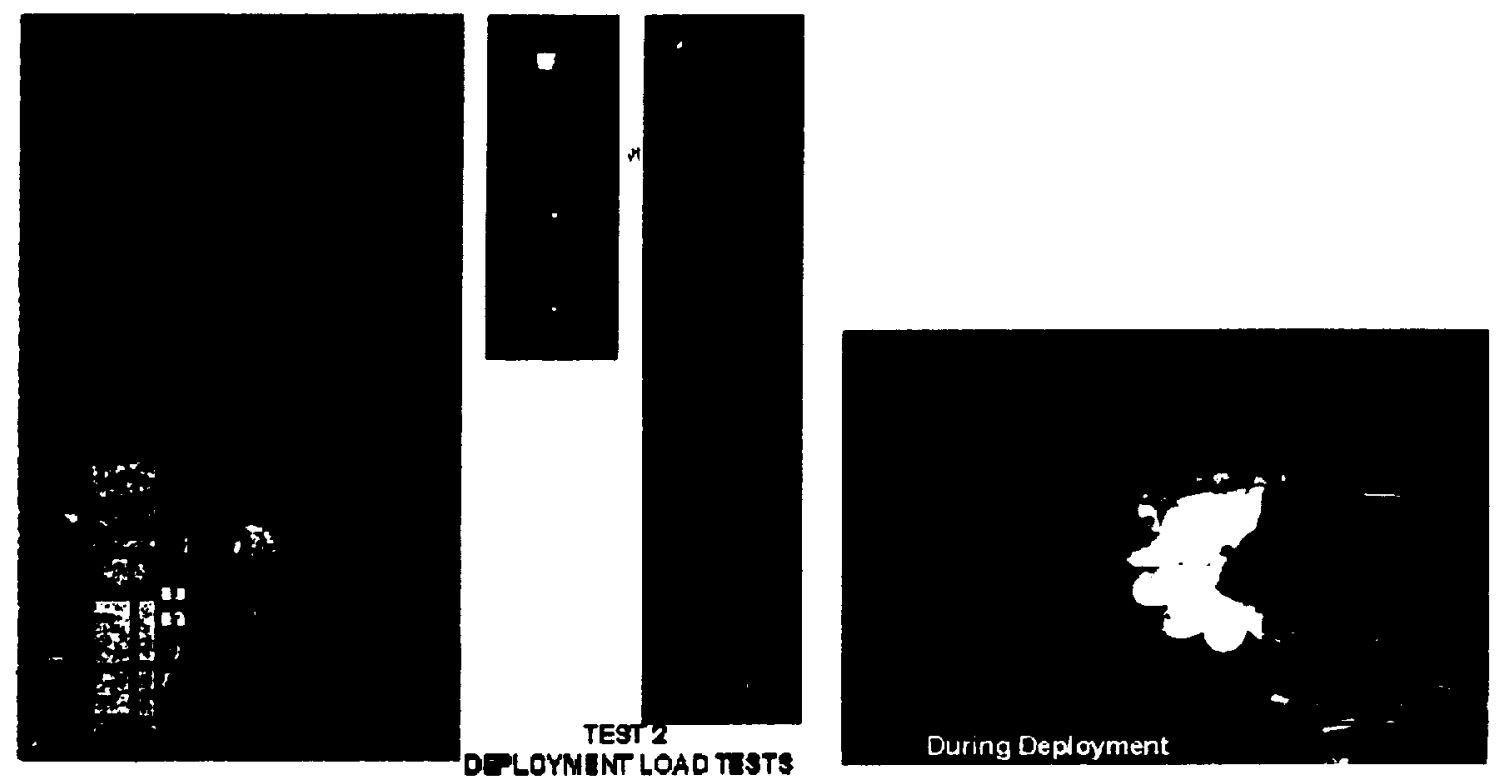

HELICONTER LIFTING PAYLOAD

Fig 5a and 5b: Fragments of the tests LAT-2 deployment view from the ground and LAT-1 deployment view from DM.

Data from the accelerometer and from the load cell measured during the deployment shown on Fig.6

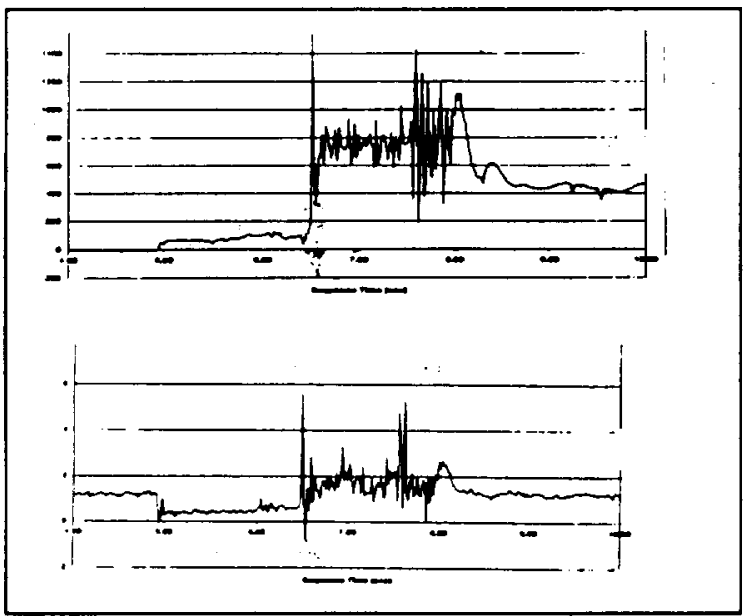

Fig.6.LAT-2 Load cell (top) and accelerometer (bottom) data.

The load cell was installed between the bottom fitting of the balloon and DM. The Pyro- 1 fired at $t=5 \mathrm{~s}$. First $1.5 \mathrm{~s}(t=5.0 \ldots 6.5 \mathrm{~s})$ balloon and DM fall almost free: that manifests in the reduced load and measured acceleration (accelerometer measures non-gravitiy acceleration). Short shock $\sim 5 \mathrm{~g}$ at $6.5 \mathrm{~s}$ is the deployment shock when the balloon is stretched. The period from $6.5 \mathrm{~s}$ to $8.0 \mathrm{~s}$ corr :sponds to tearing of the ripstitch; the average iension is $\sim 800 \mathrm{~N}(80 \mathrm{~kg})$ i.e. 'iominal force of the ripstitch

Low altituice deployment and inflution test( $L A T$ 3: The first complete flight test of the deployment and ir?ation of 0.5 ial Mylar balloon was performed August 23, 1998 over El Mirage Dry Lake, CA. A sub-scale 3-m diameter spherical balloon was used in the test. The available amount of gas was enough to inflate $35 \%$ of the maximum volume of the balloon. This test provided an opportunity to validate the use of the bottom inflation system for missions to bodies with much denser atmospheres than Mars - specifically Venus and Titan.

The flight system was suspended from the helicopter with a $300 \mathrm{~m}$ long tether to avoid effect of the downwash which could have induced undesirable rotation of the payload. The flight system was released by actuation of a hotwire cutter by the helicopter pilot. An RF command then initiated the balloon deployment sequence shortly after the parachute deployment. The solenoid valve opened and inflation started 
15 seconds after the deployment of the balloon. The valve was closed 64 seconds later when all helium was delivered into the balloon.

Fig. 7 shows the pressure at the output of gas Tanks (open squares) and at the output of the pressure regulator (black diamonds).

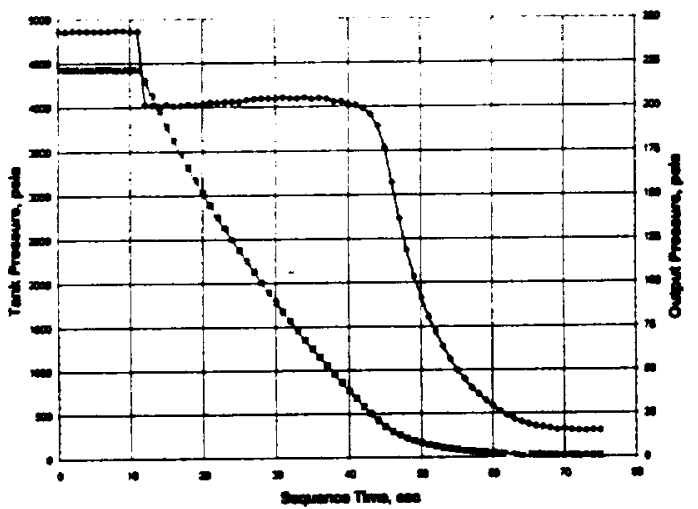

Fig.7. LAT-3 Tank pressure and tressure at the of the regulator

Inflation started at $t=11 \mathrm{~s}$. The period $11 \ldots 43 \mathrm{~s}$ corresponds to period of effective operation of the pressure regulator. This part is well approximated with a constant flow rate inflation $\sim 25 \mathrm{~g} / \mathrm{sec}$. When the tank pressure approaches the regulator level $(300 \mathrm{psi})$ the inflation continues in a blow-down mode with exponential decrease of the tani pressure

The mass of DM attached to the buttom fitting of the balıoon was $-36 \mathrm{~kg}$. The dynaniic pressure during the inflation was expected to be about 20 $\mathrm{Pa}$. The balloon was deployed and inflated successfully. In ten seconds the Pyro-3 cut the inflation hose and the recovery parachute deployed. The balloon was destroyed by a tether attached to DM to avoid a possibility of drift out of the test area.

Fig.8 shows moments of balloon deployment and of separation of DM from the inflated balloon.

The main results of the test were:

- Validation of the bottom inflation concept for the tropospheric deployment

- Validation of stability of the configuration: during the test the parachute with the attached balloon experienced a pronounced gliding and oscillations; it did not caused any aerodynamic instability and the inflation was completed successfully
- Confirmation that twisting of the halloon and significant rotation of DM are not developing during the deployment and inflation process.

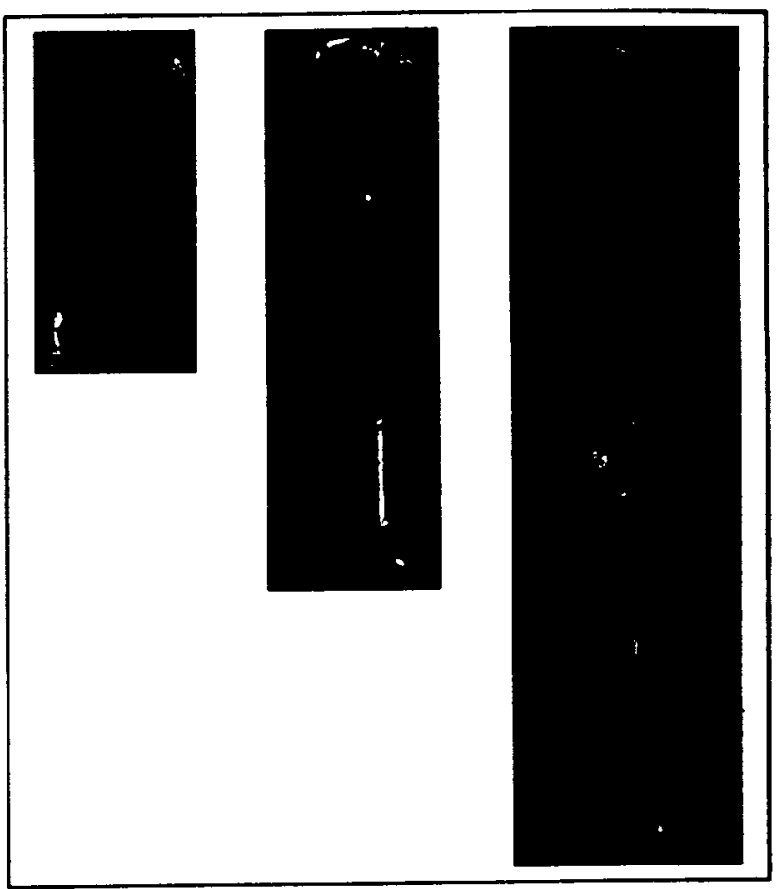

Fig.8. LAT-3 Deployment and inflation of 3-m Mylar balloon

This test has a special value since the atmospheric environment and the size of the balloon were appropriate to the deployment at the altitude $\sim 53 \mathrm{~km}$ in the atmosphere of Venus. The test validated the techrology of the deployment and inflation of a thin film balloon in the planets with dense atmospheres (Venus, Titan, and Jupiter). The VEGA balloon experience though extremely important was not completely adequate since the material of the VEGA balloon was much more robust $(300$ $\mathrm{g} / \mathrm{m} 2$ ) and a much heavier top inflation system was used.

\section{STRATOSPHERIC TESTS}

High altitude (stratospheric) tests were conducted over water in the Gulf of Mexico and from the island of Hawaii.

Flight System Design: For the stratospheric tests, some design changes were made

- Flexible fabric bag replaced the healy solid balloon container. 
- A real time video system replaced the casselte recorder because sate recovery of the test system could not be guaranteed

- The Might avionics was qualified in a thermovacuum chamber

The 10-m balloon packed into the bag was placed in the chamber also to evacuate the residual gas. It was assumed that the method of packaging will provide a pathway for the gas to the opened fitting. The pressure and temperature change in the chamber simulated the expected ascent profile. The shapes of the bag and of the balloon were continuously monitored.

The amount of the residual gas was evaluated by comparing the size of balloon after a rapid pressurization of the chamber. The balloon was contracted less than $10 \%$ in diameter when the pressure increased from $4.8 \mathrm{mb}$ to $1000 \mathrm{mb}$. It corresponds -9 I at $4.8 \mathrm{mb}$. The efficiency of the gas evacuation :vas confirmed in the stratospheric test.

The configuration of the payload was modified: only one tank was used for the inflation and the system was put into the hemispherical shell to demonstrate the possibility to meet the Micromission configuration requirements. The view of the assembled DM is shown on Fig. 9

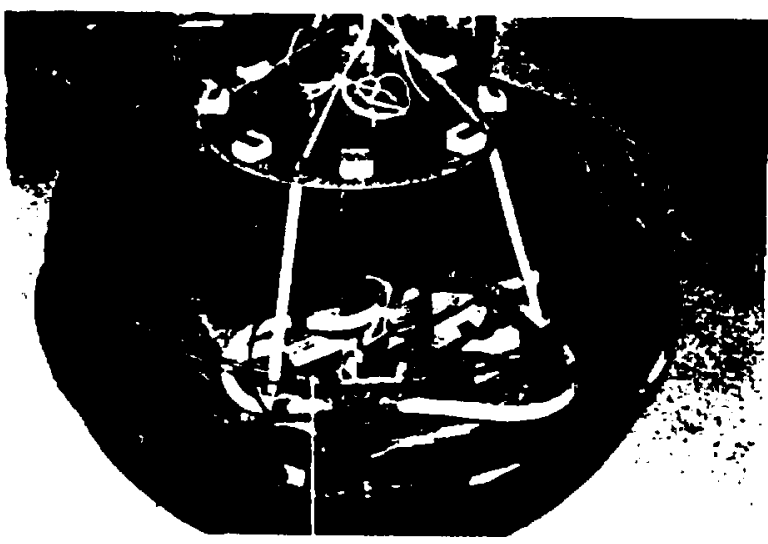

Fig.9. STRA-2 Deployment Module

The first two attempts (STRA-1 and STRA-2) fail due to failures at the carrier-balloon launch. As a result of this. we took special measures to reduce launch risks. The measures included: installation of an anemometer at the launch site and the round-clock monitoring of the local winds, a numerical model of the launch process designed to determine the maximum launch wind and several lese latumines perfiormed at JPL. sirict criteria for stare of intation and launch were adopled and the latulh procedures were revised.

Stratospheric Test at Hawaii Launch Site (STRA-2A). The launch on March 71999 was completely successful (Fig.10). The flight system reached the altitude $-34.5 \mathrm{~km}$ in $\sim 90 \mathrm{~min}$ after the launch: by this time the balloon was at $50 \mathrm{~km}$ from the launch site. Unexpectedly the command/data link that demonstrated a reliable performance in all previous tests failed just few minutes after the launch. It resulted in loss of all data except for video and radiosonde and made impossible earlier start of the test sequence.

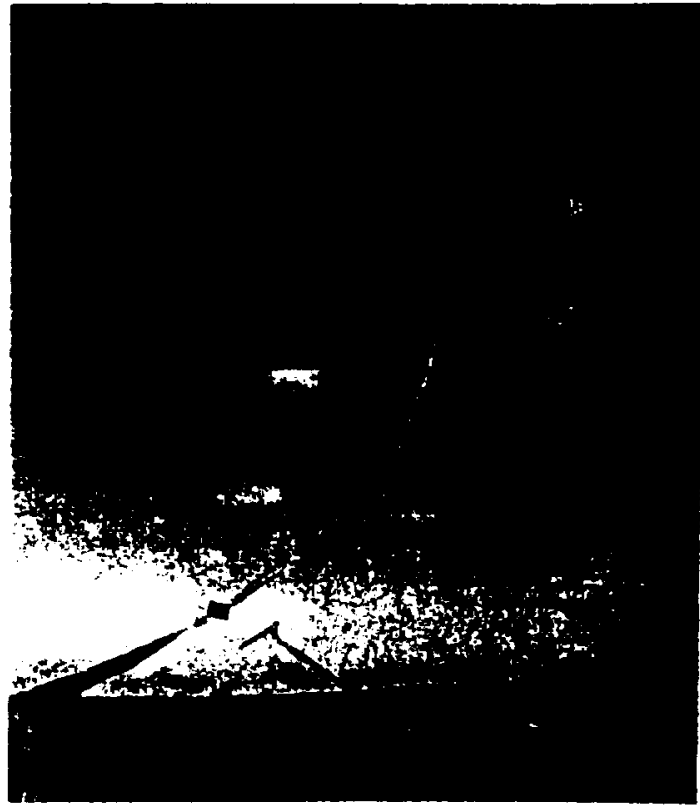

Fig 10: STRA-2A Launch

The balloon drifted for $80 \mathrm{~min}$ more when a timer in FTC initiated the preprogrammed deployment sequence. By this time the balloon was at $110-14(0 \mathrm{~km}$ from the ground station well out of the nominal range of TV system and the quality of TV data decreased dramatically.

Nominal hight ststem configurations during the phases of the stratospheric test were shown carlier on Fig.:

In spice ol poor yuality of the TV link the data irom both ammeras ( $(A . M-1$ and $(C A, H-2)$ and 
radiosonde data have permitted the reconstruction of the sequence of test events.

The first three steps of the deployment/inflation sequence occurred as planned.

- At 10:03:19 HST the Pyro-A fired by a radio-command from FTC and the parachute with DM separated from the carrier-balloon. The parachute was inflated in 2.2 seconds and the motion of the system stabilized. The descent velocity was about $40 \mathrm{~m} / \mathrm{s}$ (dynamic pressure $\sim 8 \mathrm{~Pa}$ ).

- At 10:03:35 Pyro-1 fired, DM was released, the balloon bag opened and balloon started to deploy (Fig.12).

- The balloon Jeployment was completed in less than 2.5 seconds. At 10:03:42 the solenoid valve opened and the inflation started. The view of the inflating balloon is captured on a few video fragments. At $\sim 10: 04: 03$ i.e. $\sim 21 \mathrm{~s}$ after start of inflation, the balloon failed and separated from DM being attached to the lower end of the ripstitch.

- After the failure DM started free fall and the parachute wiin attached Camera-2, ripstitch and balloon erivelope slowly descended. The video and radiosonde data from DM received for $.4 .5 \mathrm{~min}$ after beginning of the deployment sequence when $D M$ was at $\sim 10$ $\mathrm{km}$ above the ocean. The video data from the parachute received for $1 \mathrm{hr} 45 \mathrm{~min}$ after deployment until 11:50 a.m.

Fig. 11 shows the distance between the DM and

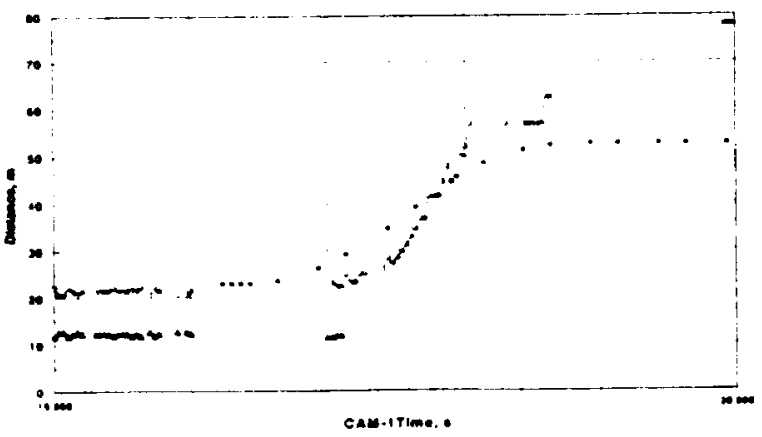

Fig 11: Distance between DM and parachute during balloon deployment the parachute during the balloon deployment estimated from analysis of images (unfilled diamonds) and the simulated values (black squares); the triangles correspond to the parachute diameter estimated from the images

The model demonstrates qualitative agreement with the results obtained from the video; the deployment process took $\sim 2$ seconds. Quantitative difference could be explained by the simplification in the model and by a poor accuracy of estimates of distance from the pictures especially when diameter of an object is small; distortion of the camera, poor quality and diffusiveness of images contribute to the errors.

Fig. 12 shows expected helium tank pressure in psia (crosses) and percentage of mass of gas delivered to balloon during the inflation (squares); the data are computed by a corresponding scaling of LAT-3 result (Fig.7)

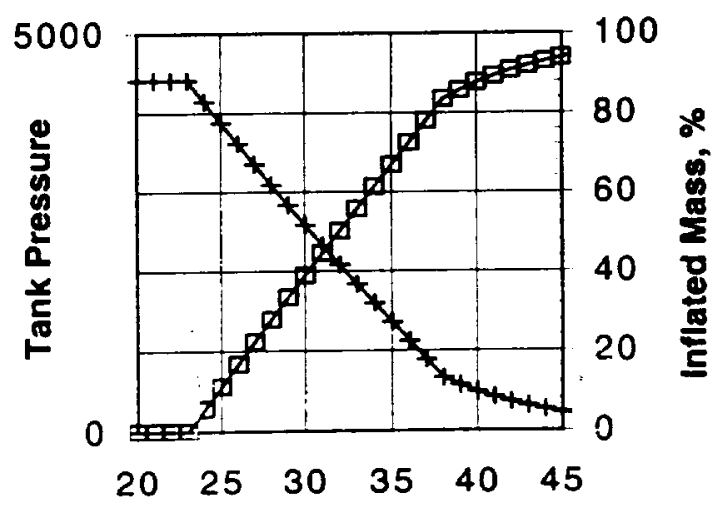

Time from Pyro-A, sec

Fig.12. Expected STRA-2A tank pressure and delivered helium mass

The data shows that by the time of the balloon failure (21 s of inflation) more than $90 \%$ of gas was delivered to the balloon. The gas occupied $-50 \%$ of the maximum balloon volume (the balloon was under the tension from the both fittings and its actual volume was less than maximum when it has a spherical shape).

The results of the tests are under analysis now; their lessons will be incorporated in the new stratospheric flight. 
In spite of that failure the major objectives of this first stratospheric test have been met and we consider the test results as highly successful.

During the test were validated: the new balloon container design; the residual gas evacuation procedure; overall concept of the flight system; system operation at stratospheric environment and under the deployment loads; the stratospheric balloon deployment without evidence of the damage; no indications on instability or twisting after deployment and at least for $90 \%$ of inflation time; Pyro-system performance

\section{SUMMARY}

The major results of the current MABVAP stage are:

- investigated the most critical factors of the airbome deployment and inflation of the light-gas thin film balloons in the planetary atmospheres;

- designed and built prototypes of the major system components of the flight system,

- developed an adequate method of packaging and residual gas evacuation,

- performed successful laboratory and flight tests at the troposphere,

- validated technology of airborne deployment of a thin-film balloons in dense atmospheres (Earth, Titan, Jupiter),

- developed capability for lcow-cost yearround stratospheric tests

- obtained encouraging results trom the first stratospheric test of a full-scale balloon

\section{Acknowledgements:}

The research described in this paper, was carried out by Jet Propulsion Laboratory, California Institute of Technology, under a contract with National Aeronautics and Space Ad́ministration. Contributions from the other members of MABVAP team are greatly acknowledged.

\section{References}

1.A.Vargas, J.Evrard, P.Maurois. Mars 96 Aerostat. - An Overview of Technology Developments and Testing. AIAA International Balloon Technology Conference, San-Francisco, CA, 1997

2.R.Zubrin, S.Price, B.Clark, J.Cantrell, R.Burke. A New MAP for Mars.Aerospace America September 1993, p.20
3.Mars 2001 Aerobot/Balloon Study (MABS). JPL Internal Document, 1996 